\title{
Intraday variability of AGNs in the southern hemisphere: the strange case of PMN J1326-5256
}

Giuseppe Cimò*

Joint Institute for VLBI in Europe

E-mail: cimodjive.nl

\section{Simon Ellingsen}

University of Tasmania

E-mail: Simon.Ellingsen@utas.edu.au

\section{Cliff Senkbail}

University of Tasmania

E-mail: cliffscutas.edu.au

\section{Peter McCulloch}

University of Tasmania

E-mail: Peter.McCulloch@utas.edu.au

\section{Hayley Bignall}

Joint Institute for VLBI in Europe

E-mail: bignallejive.nl

\section{Lucyna Kedziora-Chudczer}

Sydney University

E-mail: Lucyna.Kedziora-Chudczerdatnf.csiro.au

\begin{abstract}
Understanding of the spectral and polarimetric characteristics of rapidly scintillating blazars is fundamental in order to describe both the innermost (sub-pc) regions of these compact objects and the interstellar medium responsible for the scintillation. A multifrequency analysis of the intraday variability in PMN J1326-5256, based on the combination of Australia Telescope Compact Array observations with the data from the monitoring projects at the University of Tasmania, will be described. Some implications concerning the structure of compact radio cores and the properties of the interstellar medium will be discussed.
\end{abstract}

The 8th European VLBI Network Symposium

September 26-29, 2006

Toruń, Poland

\footnotetext{
* Speaker.

${ }^{\dagger}$ This research has been supported by the ARC grant number DP0342500 at the University of Tasmania.
} 


\section{Introduction}

Fast flux density variations on intraday time scale, i.e. intraday variability (IDV, Witzel et al, 1986 [9], Heeschen et al, 1987 [3]), are observed in some compact sources at centimetre wavelengths. If we consider these variations as intrinsic, the observed rapid fluctuations imply, via the light travel time argument, very small source sizes and very high apparent brightness temperatures (up to $10^{21} \mathrm{~K}$ ). On the other hand, such compact objects must show interstellar scintillation (ISS) due to the propagation of radio waves in the turbulent interstellar medium (ISM) of our Galaxy. Nowadays, interstellar scattering is believed to be the primary mechanics responsible for rapid variations observed at centimetre wavelengths (Jauncey et al, 2003 [5]).

The main characteristic of IDV is the variability on time scales of a day to less than one hour of both total $(S)$ and linearly polarized $(P)$ flux densities. The amplitudes of the fluctuations differ from source to source but are typically in the range $m_{S}=5-20 \%$, where $m_{S}$ is the total intensity flux density modulation index $\left(m_{S}=\sigma_{S} / S\right)$. Often, the variability is more pronounced in the polarized than in the total flux $\left(m_{P}>100 \%\right)$. Usually, the variations in $S$ and $P$ appear correlated but in some cases are anti-correlated.

An ISS origin for the IDV requires the time scales of the variations to exhibit seasonal modulations when the projected velocity of the Earth and the interstellar matter have similar speed and direction (Rickett et al, 2001 [8], Jauncey \& Macquart 2001 [4]). Therefore, a proper understanding of the IDV phenomenon needs long-term continuous monitoring of IDV sources. Using the Ceduna 30-m radio telescope of the University of Tasmania, we are continuously monitoring the variability of a number of compact sources at $6.7 \mathrm{GHz}$ in order to obtain information about the variability pattern during the course of the year (McCulloch et al, 2005 [7]). The Ceduna continuous monitoring programme is essential to collect information about the long-term characteristics of the observed flux density fluctuations.

A peculiar quiescent period has been observed in the otherwise strongly variable source PMN J1326-5256 during our Ceduna intraday variability monitoring project. We have also detected fast and pronounced polarization changes during this low-level variability phase of the total flux density using the Australia Telescope Compact Array (ATCA).

\section{Observations}

Observations of PMN J1326-5256 over the last 3 years have shown a complex variability pattern in this intraday variable source. This peculiar object has changed its scintillation behaviour since the early observations going through the following stages: fast variations - quiescence long-term variability - fast variations.

Here, we present the observations of J1326-5256 carried out with the 30-m Ceduna radio telescope at $6.7 \mathrm{GHz}$ (see fig.11). The total intensities of the target source and the calibrators (i.e. B1934-638) have been measured by means of cross-scans in both RA and Dec.

Furthermore, we present data from the observations with the ATCA carried out simultaneously at 1.6, 2.2, 4.8, and $8.4 \mathrm{GHz}$ (see fig.⿹, total intensity on the top panels, Stokes Q and U on the bottom panels). The data for both total intensity and polarisation have been reduced using the MIRIAD package. 


\section{PMN J1326-5256}
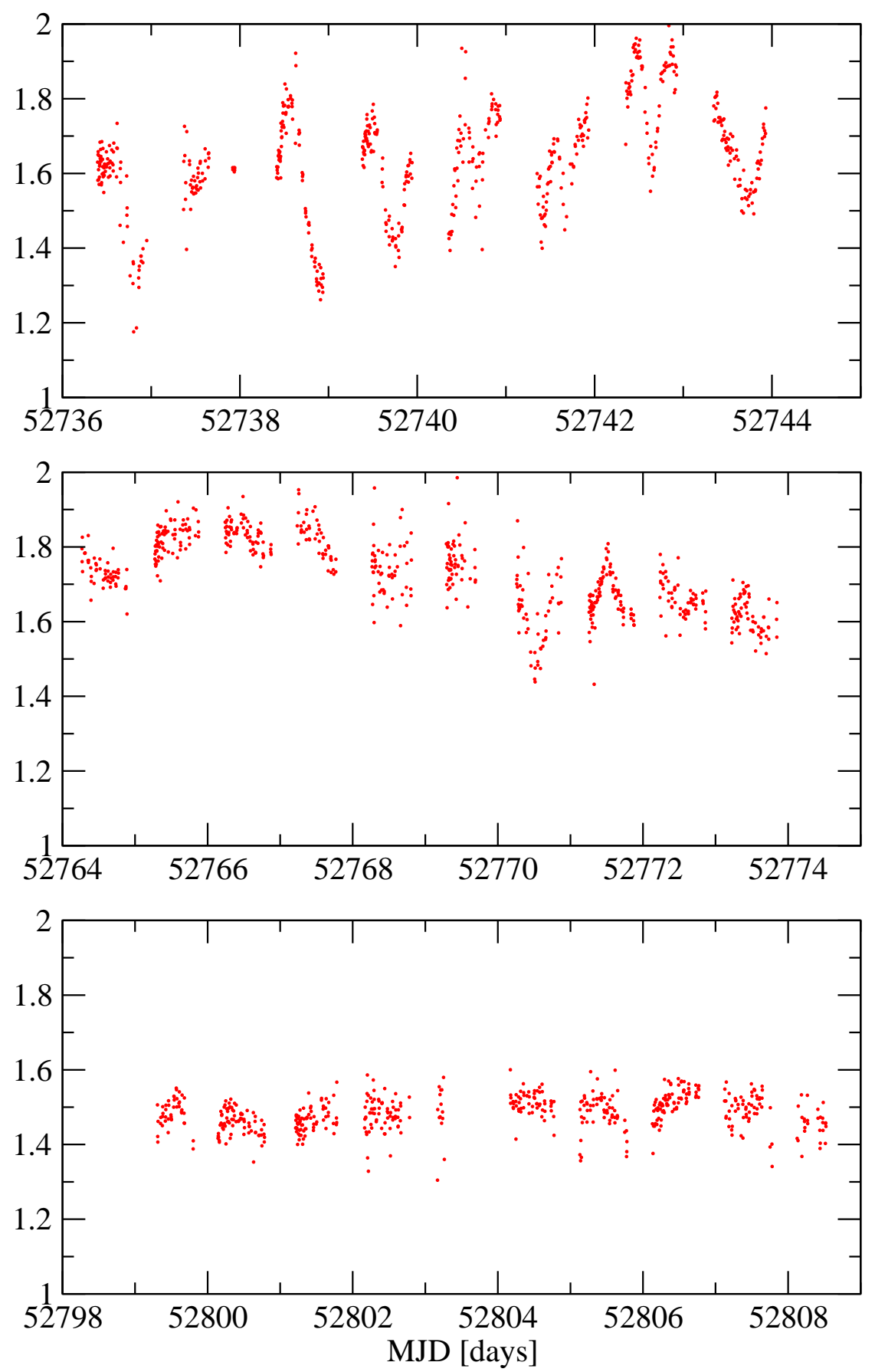

Figure 1: Intraday variations of the total flux density of PMN J1326-5256 at $6.7 \mathrm{GHz}$. Note the slow-down after MJD 52744. 


\section{Results}

Transient IDV has been observed in a number of sources. However, the peculiar behaviour of PMN J1326-5256 differs from that seen in other episodic IDV sources. For example, in B0917+624, the amplitude of the variations changed from $\sim 10 \%$ to became "stable" at a level of 1-2\% (Fuhrmann et al, 2002 [沟]), whereas in PKS 0405-385, the extreme variability lasts several months and is followed by periods of the order of the years when only slow changes are observed (Kedziora-Chudczer 2006 [6]).

In the course of the Ceduna monitoring project, PMN J1326-5256 has shown pronounced IDV with a complex pattern. Our time series analysis has revealed multiple characteristic time scales: $\tau \sim 0.4$ days and $\tau \sim 1.4$ days up to long-term (>few days) variability. PMN J1326-5256 was also observed with the ATCA during monitoring of compact radio sources at 4.8 and $8.6 \mathrm{GHz}$ (Bignall et al, 2002 [1]). In this set of observations, the variations in polarized and total flux densities were strongly correlated in early 2001, but later became uncorrelated. This implies a complex structure of the radio core.

Suddenly, in April 2003, the flux density fluctuations of this strongly variable object ceased on a time scale of less than two weeks (see fig.1). In the following months up to December 2003, the source appeared to be stable around a mean flux density of $1.5 \mathrm{Jy}$ apart from brief episodes of variability. During these sporadic events, the flux density slightly increased and decreased with time scale of $\sim 1$ day before reverting to its quiescent phase. Measurements made in January 2004 at Ceduna show an intriguing pattern: we noticed a well-defined variability although with a longer time scale (weeks) and smaller amplitude than seen before. Furthermore, the ATCA data shows multi-frequency variability of the total flux density (in agreement with the Ceduna results), but interestingly, very fast ( $\sim 2$ hour) polarization changes have also been detected (see bottom panels on fig.2).

A plausible explanation of the slow-down in the variations is that we have observed an annual modulation of the scintillation. However, such a seasonal effect can be ruled out since we know from previous observations that the source showed very strong variability in the first part of the year (Bignall et al, 2002 [1]). One could interpret this sudden change in the framework of the ISS theory as due to quenched scintillation caused by changes either in the scattering size in the ISM or in the structure of the innermost region of the source.

Variations of the electron density or change of the distance of the scattering screen could also be responsible for the cessation of the rapid variability. Alternatively, one could imagine a clumpy ISM with moving clouds at different distances. On the other hand, the emission of a new jet component can lead to an apparent source size larger than the scattering size in our Galaxy, thereby canceling out the variability. Finally, the observed complex pattern could be a mixture of scintillation (annual modulation) and source-intrinsic (i.e. pc-scale structures) effects. We have seen that the source was still scintillating in the polarized components. This suggests that these structures are smaller than the scintillating total flux density component. 

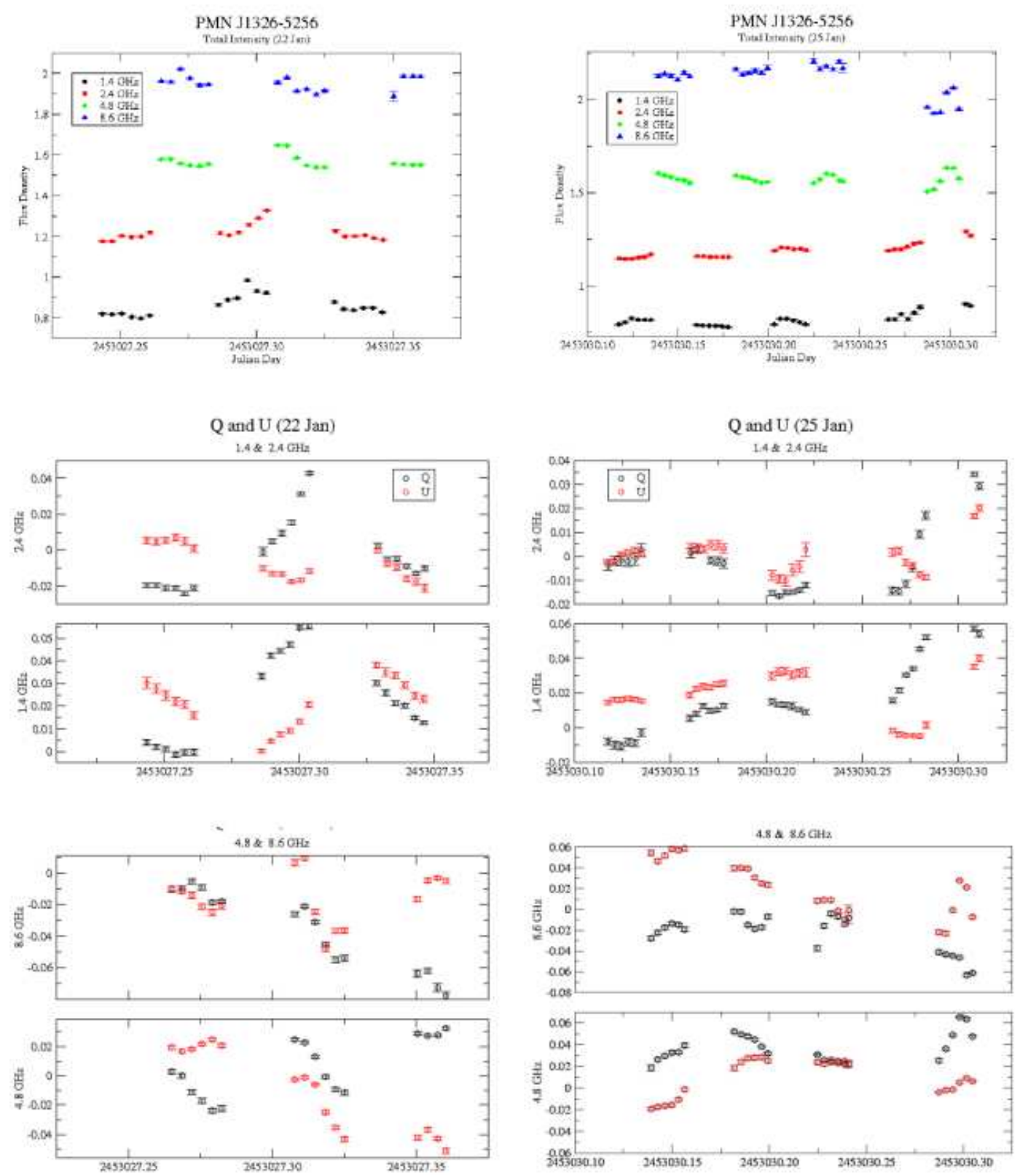

Figure 2: Intraday variations of $1326-5256$ at the ATCA in January 2004. Top two panels correspond to total intensity variations. Bottom four panels show $\mathrm{Q}$ and $\mathrm{U}$ variation. 


\section{Conclusion}

Using the Ceduna 30-m radio telescope of the University of Tasmania, we monitored the variability at $6.7 \mathrm{GHz}$ of several compact sources (McCulloch et al, 2005 [7]) in order to establish their annual cycles and variability characteristics.

When explaining the observed sudden change in the variability pattern of J1326-5256 taking into account only propagation effects, changes in the mean properties of the ionized ISM along the line of sight should be fully responsible for the observed cessation of variability. However, one should expect also a slow-down in both Stokes Q and U, which is not the case. Therefore, a scenario, which also takes into account the source structure at micro-arcsecond scale, seems to be more plausible in the case of PMN J1326-5256. We suggest that the source scintillation pattern has changed due to an intrinsic "long-term" change in the compact core. An observable effect of a multi-component scattered core at micro-arcsecond scale is the complex polarization scintillation pattern. Polarization variability is usually faster and stronger and can be detected also during quiescent phases of the total flux density fluctuations. This is due to the smaller sizes of the polarised components. Accordingly, we have detected fast ( $\sim 2$ hour) polarization changes during our ATCA experiment in late January 2004. Only the component responsible for the total intensity variations has become large enough to "quench" the scattering while we still observe the fast scintillating polarized subcomponents in the jet.

\section{References}

[1] Bignall H. E., Jauncey D. L., Kedziora-Chudczer L. L. et al. 2002, New Results from an ATCA Study of Intraday Variable Radio Sources, PASA 19, 29

[2] Fuhrmann L., Krichbaum T. P., Cimò G. et al. 2002, Annual Modulation in the Variability Properties of the IDV Source 0917+624?, PASA 19, 64

[3] Heeschen D. S. Krichbaum T. P, Schalinski C. J. \& Witzel A, 1987, Rapid variability of extragalactic radio sources, AJ 94, 1493

[4] Jauncey D. L. \& Macquart J.-P., Intra-day variability and the interstellar medium towards 0917+624, 2001, A\&A 370, L9

[5] Jauncey D. L., Bignall H. E., Lovell J. E. J. et al. 2003, Interstellar Scintillation and Radio Intra-Day Variability in "Radio Astronomy at the Fringe", Eds Zensus, Cohen \& Ros, PASP Vol 300, p 199

[6] Kedziora-Chudczer, L. 2006, Long-term monitoring of the intra-day variable quasar PKS 0405-385, MNRAS 323, 1411

[7] McCulloch P. M., Ellingsen S. P., Jauncey D. L. et al. 2005, COSMIC: Microarcsecond Resolution with a 30 Meter Radio Telescope, AJ 129, 2034

[8] Rickett B. J., Witzel A., Kraus A. et al. 2001, Annual Modulation in the Intraday Variability of Quasar 0917+624 due to Interstellar Scintillation, ApJ 550L, 11

[9] Witzel A., Heeschen D. S., Schalinski C. J \& Krichbaum T. P, 1986, Kurzzeit-Variabilität extragalaktischer Radioquellen, Mitt. Astron. Ges 65, 239 